\title{
Torsion exponents in stable homotopy and the Hurewicz homomorphism
}

\author{
AKHIL MATHEW
}

\begin{abstract}
We give estimates for the torsion in the Postnikov sections $\tau_{[1, n]} S^{0}$ of the sphere spectrum, and we show that the $p$-localization is annihilated by $p^{n /(2 p-2)+O(1)}$. This leads to explicit bounds on the exponents of the kernel and cokernel of the Hurewicz map $\pi_{*}(X) \rightarrow H_{*}(X ; \mathbb{Z})$ for a connective spectrum $X$. Such bounds were first considered by Arlettaz, although our estimates are tighter, and we prove that they are the best possible up to a constant factor. As applications, we sharpen existing bounds on the orders of $k$-invariants in a connective spectrum, sharpen bounds on the unstable Hurewicz map of an infinite loop space, and prove an exponent theorem for the equivariant stable stems.
\end{abstract}

$55 \mathrm{P} 42,55 \mathrm{Q} 10$

\section{Introduction}

Let $X$ be a spectrum. Then there is a natural map (the Hurewicz map) of graded abelian groups

$$
\pi_{*}(X) \rightarrow H_{*}(X ; \mathbb{Z})
$$

which is an isomorphism rationally. In general, this is the best that one can say. For instance, given an element $x \in \pi_{n}(X)$ annihilated by the Hurewicz map, we know that $x$ is torsion, but we cannot a priori give an integer $m$ such that $m x=0$. For example, if $K$ denotes periodic complex $K$-theory, then $K / p^{k}$ has trivial homology for each $k$, but it contains elements in homotopy of order $p^{k}$.

If, however, $X$ is connective, then one can do better. For instance, the Hurewicz theorem states in this case that the map $\pi_{0}(X) \rightarrow H_{0}(X ; \mathbb{Z})$ is an isomorphism. The map $\pi_{1}(X) \rightarrow H_{1}(X ; \mathbb{Z})$ need not be an isomorphism, but it is surjective, and any element in the kernel must be annihilated by 2 . There is a formal argument that, in any degree, "universal" bounds must exist.

Proposition 1.1 There exists a function $M: \mathbb{Z}_{\geq 0} \rightarrow \mathbb{Z}_{>0}$ with the following property: if $X$ is any connective spectrum, then the kernel and cokernel of the Hurewicz map $\pi_{n}(X) \rightarrow H_{n}(X ; \mathbb{Z})$ are annihilated by $M(n)$. 
Proof We consider the case of the kernel; the other case is similar. Suppose there existed no such function. Then, there exists an integer $n$ and connective spectra $X_{1}, X_{2}, \ldots$ together with elements $x_{i} \in \pi_{n}\left(X_{i}\right)$ for each $i$ such that:

(a) Each $x_{i}$ is in the kernel of the Hurewicz map (and thus torsion).

(b) The orders of the $x_{i}$ are unbounded.

In this case, we can form a connective spectrum $X=\prod_{i=1}^{\infty} X_{i}$. Since homology commutes with arbitrary products for connective spectra, as $H \mathbb{Z}$ can be given a cell decomposition with finitely many cells in each degree (see [2, Theorem 15.2, part III]), it follows that we obtain an element $x=\left(x_{i}\right)_{i \geq 1} \in \pi_{n}(X)=\prod_{i \geq 1} \pi_{n}\left(X_{i}\right)$ which is annihilated by the Hurewicz map. However, $x$ cannot be torsion since the orders of the $x_{i}$ are unbounded.

We note that the above argument is very general. For instance, it shows that the nilpotence theorem [12] implies that there exists a universal function $P(n): \mathbb{Z}_{\geq 0} \rightarrow \mathbb{Z}_{>0}$ such that if $R$ is a connective ring spectrum and $x \in \pi_{n}(R)$ is annihilated by the $M U-$ Hurewicz map, then $x^{P(n)}=0$. The determination of the best possible function $P(n)$ is closely related to the questions raised by Hopkins in [15].

Proposition 1.1 appears in [6], where an upper bound for the universal function $M(n)$ is established (although the above argument may be older).

Theorem 1.2 (Arlettaz [6, Theorem 4.1]) If $X$ is any connective spectrum, then the kernel of $\pi_{n}(X) \rightarrow H_{n}(X ; \mathbb{Z})$ is annihilated by $\rho_{1} \cdots \rho_{n}$, where $\rho_{i}$ is the smallest positive integer that annihilates the torsion group $\pi_{i}\left(S^{0}\right)$. The cokernel is annihilated by $\rho_{1} \cdots \rho_{n-1}$.

Different variants of this result have appeared in [5; 8], and this result has also been discussed in [10]. The purpose of this note is to find the best possible bounds for these torsion exponents, up to small constants. We will do so at each prime $p$. In particular, we prove:

Theorem 1.3 Let $X$ be a connective spectrum and let $n>0$.

(a) The 2-exponent of the kernel of the Hurewicz map $\pi_{n}(X) \rightarrow H_{n}(X ; \mathbb{Z})$ is at most $\lceil n / 2\rceil+3$ : that is, $2^{\lceil n / 2\rceil+3}$ annihilates the 2-part of the kernel.

(b) If $p$ is an odd prime, the $p$-exponent of the kernel of the Hurewicz map $\pi_{n}(X) \rightarrow H_{n}(X ; \mathbb{Z})$ is at most $\lceil(n+3) /(2 p-2)\rceil+1$.

(c) The 2-exponent of the cokernel of the Hurewicz map is at most $\lceil(n-1) / 2\rceil+3$.

(d) If $p$ is an odd prime, the $p$-exponent of the cokernel of the Hurewicz map is at most $\lceil(n+2) /(2 p-2)\rceil+1$. 
We will also show that these bounds are close to being the best possible.

Proposition 1.4 (a) For each $r$, there exists a connective 2-local spectrum $X$ and an element $x \in \pi_{2 r-1}(X)$ in the kernel of the Hurewicz map such that the order of $x$ is at least $2^{r-1}$.

(b) Let $p$ be an odd prime. For each $r$, there exists a connective $p$-local spectrum $X$ and an element $x \in \pi_{(2 p-2) r+1}(X)$ annihilated by the Hurewicz map such that the order of $x$ is at least $p^{r}$.

Our strategy in proving Theorem 1.3 is to translate the above question into one about the Postnikov sections $\tau_{[1, n]} S^{0}$ and their exponents in the homotopy category of spectra (rather than the exponents of some algebraic invariant). We shall use a classical technique with vanishing lines to show that, at a prime $p$, the $\tau_{[1, n]} S^{0}$ are annihilated by $p^{n /(2 p-2)+O(1)}$. This, combined with a bit of diagram-chasing, will imply the upper bound of Theorem 1.3. The lower bounds will follow from explicit examples.

Finally, we show that these methods have additional applications and that the precise order of the $n$-truncations $\tau_{[1, n]} S^{0}$ play an important role in several settings. For instance, we sharpen bounds of Arlettaz [4] on the orders of the $k$-invariants of a spectrum in Corollary 6.2, improve and make explicit half of a result of Beilinson [10] on the (unstable) Hurewicz map $\pi_{n}(X) \rightarrow H_{n}(X ; \mathbb{Z})$ for $X$ an infinite loop space in Theorem 6.3, and prove an exponent theorem for the equivariant stable stems in Theorem 6.6.

We also obtain, as a consequence, the following result.

Theorem 1.5 Let $p$ be a prime number. Let $X$ be a spectrum with homotopy groups concentrated in degrees $[a, b]$. Suppose each $\pi_{i}(X)$ is annihilated by $p^{k}$. Then $p^{k+(b-a) /(p-1)+8}$ annihilates $X$ (see Definition 2.1 below).

We have not tried to make the bounds in Theorem 1.5 as sharp as possible since we suspect that our techniques are not sharp to begin with.

Notation In this paper, for a spectrum $X$, we will write $\tau_{[a, b]} X$ to denote the Postnikov section of $X$ with homotopy groups in the range $[a, b]$, ie $\tau_{\geq b} \tau_{\leq a} X$. Given spectra $X$ and $Y$, we will let $\operatorname{Hom}(X, Y)$ denote the function spectrum from $X$ into $Y$, so $\pi_{0} \operatorname{Hom}(X, Y)$ denotes homotopy classes of maps $X \rightarrow Y$.

Acknowledgments I would like to thank Mike Hopkins and Haynes Miller, from whom (and whose papers) I learned many of the ideas used here. I would also like to thank Peter May for several helpful comments and Dustin Clausen for pointing me to [10]. The author was supported by the NSF Graduate Fellowship under grant DGE-110640. 


\section{Definitions}

Let $\mathcal{C}$ be a triangulated category. We recall:

Definition 2.1 Let $X \in \mathcal{C}$ be an object. We will say that $X$ is annihilated by $n \in \mathbb{Z}_{>0}$ if $n \operatorname{id}_{X} \in \operatorname{Hom}_{\mathcal{C}}(X, X)$ is equal to zero. We let $\exp (X)$ denote the minimal $n$ (or $\infty$ if none exists) such that $n$ annihilates $X$.

Let $\mathcal{D}$ be any additive category and $F: \mathcal{C} \rightarrow \mathcal{D}$ any additive functor. If $X \in \mathcal{C}$ is annihilated by $n$, then $F(X) \in \mathcal{D}$ has $n \operatorname{id}_{F(X)}=0$, too. Here are several important examples of this phenomenon.

Example 2.2 Given any (co)homological functor $F: \mathcal{C} \rightarrow \mathrm{Ab}$, the value of $F$ on an object annihilated by $n$ is a torsion abelian group of exponent at most $n$. For instance, if $X$ is a spectrum annihilated by $n$, then the homotopy groups of $X$ all have exponent at most $n$.

Example 2.3 Suppose $\mathcal{C}$ has a $t$-structure, so that we can construct truncation functors $\tau_{\leq k}: \mathcal{C} \rightarrow \mathcal{C}$ for $k \in \mathbb{Z}$. Let $X \in \mathcal{C}$ be any object. Then, for any $k, \exp \left(\tau_{\leq k} X\right) \mid \exp (X)$.

Example 2.4 Suppose $\mathcal{C}$ has a compatible monoidal structure $\wedge$. Then if $X, Y \in \mathcal{C}$, we have $\exp (X \wedge Y) \mid \operatorname{gcd}(\exp (X), \exp (Y))$.

Next, we note that such torsion questions can be reduced to local ones at each prime $p$, and it will be therefore convenient to have the following notation.

Definition 2.5 Given $X \in \mathcal{C}$, we define $\exp _{p}(X)$ to be the minimal integer $n \geq 0$ (or $\infty$ if none exists) such that $p^{n} \mathrm{id}_{X}=0$ in the group $\operatorname{Hom}_{\mathcal{C}}(X, X)_{(p)}$. For a torsion abelian group $A$, we will also use the notation $\exp _{p}(A)$ in this manner.

Proposition 2.6 Let $X^{\prime} \rightarrow X \rightarrow X^{\prime \prime}$ be a cofiber sequence in $\mathcal{C}$. Suppose $X^{\prime}$ is annihilated by $m$ and $X^{\prime \prime}$ is annihilated by $n$. Then $X$ is annihilated by $m n$. Equivalently, $\exp _{p}(X) \leq \exp _{p}\left(X^{\prime}\right)+\exp _{p}\left(X^{\prime \prime}\right)$ for each prime $p$.

Proof We have an exact sequence of abelian groups

$$
\operatorname{Hom}_{\mathcal{C}}\left(X, X^{\prime}\right) \rightarrow \operatorname{Hom}_{\mathcal{C}}(X, X) \rightarrow \operatorname{Hom}_{\mathcal{C}}\left(X, X^{\prime \prime}\right) .
$$

If $X^{\prime}$ and $X^{\prime \prime}$ are annihilated by $m$ and $n$, respectively, then it follows that groups on the edges of the above exact sequence are of exponents dividing $m$ and $n$, respectively. It follows that $\operatorname{Hom}_{\mathcal{C}}(X, X)$ is annihilated by $m n$, and in particular, the identity map $\operatorname{id}_{X} \in \operatorname{Hom}_{\mathcal{C}}(X, X)$ is annihilated by $m n$. 
Corollary 2.7 Let $X$ be a spectrum with homotopy groups concentrated in degrees $[m, n]$ for $m, n \in \mathbb{Z}$. Suppose for each $i \in[m, n]$, we have an integer $e_{i}>0$ with $e_{i} \pi_{i}(X)=0$. Then $\exp (X) \mid \prod_{i=m}^{n} e_{i}$.

The main purpose of this paper is to determine the behavior, as $n$ varies, of the function $\exp _{p}\left(\tau_{[1, n]} S^{0}\right)$. Corollary 2.7 gives the bound that $\exp _{p}\left(\tau_{[1, n]} S^{0}\right)$ is at most the sum of the exponents of the torsion abelian groups $\pi_{i}\left(S^{0}\right)_{(p)}$ for $1 \leq i \leq n$. We will give a stronger upper bound for this function, and show that it is essentially optimal.

Theorem 2.8 (Main theorem) (a) Let $p=2$. Then

$$
\left\lfloor\frac{n-1}{2}\right\rfloor \leq \exp _{2}\left(\tau_{[1, n]} S^{0}\right) \leq\left\lceil\frac{n}{2}\right\rceil+3 .
$$

(b) Let $p$ be odd. Then

$$
\left\lfloor\frac{n-1}{2 p-2}\right\rfloor \leq \exp _{p}\left(\tau_{[1, n]} S^{0}\right) \leq\left\lceil\frac{n+3}{2 p-2}\right\rceil+1 .
$$

The upper bounds will be proved in Proposition 3.4 below, and the lower bounds will be proved in Propositions 4.2 and 4.3. They include, as a special case, estimates on the exponents on the homotopy groups of $S^{0}$, which were classically known (and in fact our method is a refinement of the proof of those estimates). Note that the exponents in the unstable homotopy groups have been studied extensively, including the precise determination at odd primes [11], and that the method of using the Adams spectral sequence to obtain such quantitative bounds has also been used by Henn [14].

\section{Upper bounds}

Let $p$ be a prime number. Let $\mathcal{A}_{p}$ denote the mod $p$ Steenrod algebra; it is a graded algebra. Recall that if $X$ is a spectrum, then the $\bmod p$ cohomology $H^{*}\left(X ; \mathbb{F}_{p}\right)$ is a graded module over $\mathcal{A}_{p}$. Our approach to the upper bounds will be based on vanishing lines in the cohomology.

Definition 3.1 Given a nonnegatively graded $\mathcal{A}_{p}$-module $M$, we will say that a function $f: \mathbb{Z}_{\geq 0} \rightarrow \mathbb{Z}_{\geq 0}$ is a vanishing function for $M$ if for all $s, t \in \mathbb{Z}_{\geq 0}$,

$$
\operatorname{Ext}_{\mathcal{A}_{p}}^{s, t}\left(M, \mathbb{F}_{2}\right)=0 \quad \text { if } t<f(s) .
$$

Recall here that $s$ is the homological degree and $t$ is the grading.

Our main technical result is the following: 
Proposition 3.2 Suppose $X$ is a connective spectrum such that each $\pi_{i}(X)$ is a finite $p$-group. Suppose the $\mathcal{A}_{p}$-module $H^{*}\left(X ; \mathbb{F}_{p}\right)$ has a vanishing function $f$. Let $n$ be an integer and let $m$ be an integer such that $f(m)-m>n$. Then $\exp _{p}\left(\tau_{[0, n]} X\right) \leq m$.

Proof Choose a minimal resolution (see, eg [19, Definition 9.3]) of $H^{*}\left(X ; \mathbb{F}_{p}\right)$ by free, graded $\mathcal{A}_{p}$-modules

$$
\cdots \rightarrow P_{1} \rightarrow P_{0} \rightarrow H^{*}\left(X ; \mathbb{F}_{p}\right) \rightarrow 0 .
$$

Here we have $\operatorname{Ext}^{s, t}\left(H^{*}\left(X ; \mathbb{F}_{p}\right), \mathbb{F}_{p}\right) \simeq \operatorname{Hom}_{\mathcal{A}_{p}}\left(P_{s}, \Sigma^{t} \mathbb{F}_{p}\right)$ by [19, Proposition 9.4]. That is, the free generators of the $P_{s}$ give precisely a basis for $\operatorname{Ext}^{s, *}\left(H^{*}\left(X ; \mathbb{F}_{p}\right) ; \mathbb{F}_{p}\right)$.

We can realize the resolution (3) topologically (see eg [19, Section 9.3]) via an Adams resolution. That is, we can find (working by induction) a tower of spectra,

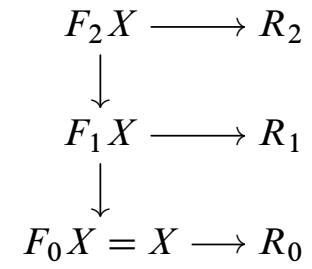

such that:

(a) Each $R_{i}$ is a wedge of copies of shifts of $H \mathbb{F}_{p}$.

(b) Each triangle $F_{i+1} X \rightarrow F_{i} X \rightarrow R_{i}$ is a cofiber sequence.

(c) The sequence of spectra

$$
X \rightarrow R_{0} \rightarrow \Sigma R_{1} \rightarrow \Sigma^{2} R_{2} \rightarrow \cdots
$$

realizes, on cohomology, the complex (3).

As a result, we find inductively that

$$
H^{*}\left(F_{i} X ; \mathbb{F}_{p}\right) \simeq \Sigma^{-i} \operatorname{im}\left(P_{i} \rightarrow P_{i-1}\right) .
$$

Now the graded $\mathcal{A}_{p}$-module $P_{i}$ is concentrated in degrees $f(i)$ and up, by hypothesis and minimality. In particular, it follows that $F_{i} X$ is $(f(i)-i)$-connective. Specifically, it follows that the map

$$
X \rightarrow \operatorname{cofib}\left(F_{i} X \rightarrow X\right)
$$

is an isomorphism on homotopy groups below $f(i)-i$. 
Finally, we observe that the cofiber of each $F_{i} X \rightarrow F_{i-1} X$ is annihilated by $p$ as it is a wedge of shifts of $H \mathbb{F}_{p}$. It follows by the octahedral axiom of triangulated categories, induction on $i$, and Proposition 2.6 that the cofiber of $F_{i} X \rightarrow F_{0} X=X$ is annihilated by $p^{i}$. Taking $i=m$, we get the claim since $\tau_{\leq n} X \simeq \tau_{\leq n}\left(\operatorname{cofib}\left(F_{m} X \rightarrow X\right)\right)$ is therefore annihilated by $p^{m}$ by Example 2.3.

Since $\mathcal{A}_{p}$ is a connected graded algebra, it follows easily (via a minimal resolution) that if $M$ is a connected graded $\mathcal{A}_{p}$-module, then $\operatorname{Ext}^{s, t}\left(M, \mathbb{F}_{p}\right)=0$ if $t<s$. Of course, this bound is too weak to help with Proposition 3.2. In fact, an integer $m$ satisfying the desired conditions will not exist if we use this bound.

We now specialize to the case of interest. Consider $\tau_{\geq 1} S^{0}=\tau_{[1, \infty]} S^{0}$. It fits into a cofiber sequence

$$
S^{0} \rightarrow H \mathbb{Z} \rightarrow \Sigma \tau_{\geq 1} S^{0}
$$

which leads to an exact sequence

$$
0 \rightarrow H^{*}\left(\Sigma \tau_{\geq 1} S^{0} ; \mathbb{F}_{p}\right) \rightarrow H^{*}\left(H \mathbb{Z} ; \mathbb{F}_{p}\right) \rightarrow H^{*}\left(S^{0} ; \mathbb{F}_{p}\right) \rightarrow 0 .
$$

We know that $\operatorname{Ext}_{\mathcal{A}_{p}}^{s, t}\left(H^{*}\left(H \mathbb{Z} ; \mathbb{F}_{p}\right) ; \mathbb{F}_{p}\right)$ vanishes unless $s=t$ (by the change-of-rings theorem [19, Fact 3, page 438]), and is one-dimensional if $s=t$; in this case, it maps isomorphically to $\operatorname{Ext}_{\mathcal{A}_{p}}^{s, s}\left(\mathbb{F}_{p}, \mathbb{F}_{p}\right)$. It follows that

$$
\operatorname{Ext}_{\mathcal{A}_{p}}^{s, t}\left(H^{*}\left(\tau_{\geq 1} S^{0} ; \mathbb{F}_{p}\right) ; \mathbb{F}_{p}\right)= \begin{cases}\operatorname{Ext}_{\mathcal{A}_{p}}^{s-1, t-1}\left(\mathbb{F}_{p} ; \mathbb{F}_{p}\right) & \text { if } s \neq t \\ 0 & \text { if } s=t\end{cases}
$$

We will need certain classical facts, due to Adams [1] at $p=2$ and Liulevicius [17] for $p>2$, about vanishing lines in the classical Adams spectral sequence. A convenient reference is [19].

\section{Proposition 3.3 [19, Theorem 9.43]}

(a) $\operatorname{Ext}_{\mathcal{A}_{2}}^{s, t}\left(\mathbb{F}_{2}, \mathbb{F}_{2}\right)=0$ for $0<s<t<3 s-3$.

(b) $\operatorname{Ext}_{\mathcal{A}_{p}}^{s, t}\left(\mathbb{F}_{p}, \mathbb{F}_{p}\right)=0$ for $0<s<t<(2 p-1) s-2$.

Note also that $\operatorname{Ext}_{\mathcal{A}_{p}}^{s, t}\left(\mathbb{F}_{p}, \mathbb{F}_{p}\right)=0$ for $t<s$. As a result, one finds that the cohomology of $\tau_{\geq 1} S^{0}$, when displayed using Adams indexing with $t-s$ on the $x$-axis and $s$ on the $y$-axis, vanishes above a line with slope $1 /(2 p-2)$.

Finally, we can prove our upper bounds.

Proposition 3.4 (a) For $p=2$, $\exp _{2}\left(\tau_{[1, n]} S^{0}\right) \leq\lceil n / 2\rceil+3$.

(b) For $p$ odd, $\exp _{p}\left(\tau_{[1, n]} S^{0}\right) \leq\lceil(n+3) /(2 p-2)\rceil+1$. 
Proof This is now a consequence of the preceding discussion. We just need to put things together.

At the prime 2, by Proposition 3.3 and (5), the $\mathcal{A}_{2}$-module $H^{*}\left(\tau_{\geq 1} S^{0} ; \mathbb{F}_{2}\right)$ has vanishing function $f(s)=3 s-5$. By Proposition 3.2, it follows that if $2 m-5>n$, then $\exp _{2}\left(\tau_{[1, n]} S^{0}\right) \leq m$. Choosing $m=\lceil n / 2\rceil+3$ gives the minimal choice.

At an odd prime, one similarly sees that $f(s)=(2 p-1) s-2 p$ is a vanishing function by Proposition 3.3 and (5). That is, if $(2 p-2) m-2 p>n$, then we have $\exp _{p}\left(\tau_{[1, n]} S^{0}\right) \leq m$. Rearranging gives the desired claim.

\section{Lower bounds}

The purpose of this section is to prove the lower bounds of Theorem 2.8. The proof of the lower bounds is completely different from the proof of the upper bounds. Namely, we will write down finite complexes that have homology annihilated by $p$ but for which the $p$-exponent grows linearly. These complexes are simply the skeleta of $B \mathbb{Z} / p$. We will show, however, that the $p$-exponent of the spectra grows linearly by looking at the complex $K$-theory. First, we need a lemma.

Lemma 4.1 Let $X$ be a finite torsion complex with cells in degrees 0 through $m$. Then, for each $p, \exp _{p}(X)=\exp _{p}\left(\tau_{[0, m]} S^{0} \wedge X\right)$.

Proof Without loss of generality, suppose that $X$ is $p$-local. We know that $\exp _{p}(X) \geq$ $\exp _{p}\left(\tau_{[0, m]} S^{0} \wedge X\right)$; see Example 2.4. Thus, we need to prove the other inequality.

Let $k=\exp _{p}(X)$. Let $\operatorname{Hom}(X, X)$ denote the endomorphism ring spectrum of $X$. The identity map $X \rightarrow X$ defines a class in $\pi_{0} \operatorname{Hom}(X, X)$, which maps isomorphically to $\pi_{0} \operatorname{Hom}\left(X, \tau_{[0, m]} S^{0} \wedge X\right)$ by the hypothesis on the cells of $X$. Thus, there exists a class in $\pi_{0} \operatorname{Hom}\left(X, \tau_{[0, m]} S^{0} \wedge X\right)$ of order exactly $p^{k}$. It follows that $\exp _{p}\left(\tau_{[0, m]} S^{0} \wedge X\right) \geq$ $k$ as desired.

We are now ready to prove our lower bound at the prime two.

Proposition 4.2 We have $\exp _{2}\left(\tau_{[1, n]} S^{0}\right) \geq\lfloor(n-1) / 2\rfloor$.

Proof Since the function $n \mapsto \exp _{2}\left(\tau_{[1, n]} S^{0}\right)$ is increasing in $n$ (see Example 2.3), it suffices to assume $n=2 r-1$ is odd. Consider the space $\mathbb{R P}^{2 r}$ (for $r \in \mathbb{Z}_{>0}$ ) and its reduced suspension spectrum $\Sigma^{\infty} \mathbb{R P}^{2 r}$, which is 2 -power torsion. We know that $\widetilde{K}^{0}\left(\mathbb{R} \mathbb{P}^{2 r}\right) \simeq \mathbb{Z} / 2^{r}$ by [9, Proposition 2.7.7]. It follows that (see Example 2.2)

$$
\exp _{2}\left(\Sigma^{\infty} \mathbb{R P}^{2 r}\right) \geq r
$$


Now $\Sigma^{\infty} \mathbb{R P}^{2 r}$ has cells in degrees 1 to $2 r$. By Lemma 4.1, $\exp _{2}\left(\tau_{[0,2 r-1]} S^{0} \wedge\right.$ $\left.\Sigma^{\infty} \mathbb{R} \mathbb{P}^{2 r}\right) \geq r$, too.

We have a cofiber sequence

$$
\tau_{[1,2 r-1]} S^{0} \wedge \Sigma^{\infty} \mathbb{R P}^{2 r} \longrightarrow \tau_{[0,2 r-1]} S^{0} \wedge \Sigma^{\infty} \mathbb{R P}^{2 r} \longrightarrow H \mathbb{Z} \wedge \Sigma^{\infty} \mathbb{R P}^{2 r}
$$

The integral homology of $\Sigma^{\infty} \mathbb{R P}^{2 r}$ is annihilated by 2 , so the $H \mathbb{Z}$-module spectrum $H \mathbb{Z} \wedge \mathbb{R P}^{2 r}$ is a wedge of copies of $H \mathbb{Z} / 2$ and is thus annihilated by 2 . It therefore follows from this cofiber sequence and Proposition 2.6 that

$$
\exp _{2}\left(\tau_{[1,2 r-1]} S^{0} \wedge \Sigma^{\infty} \mathbb{R P}^{2 r}\right) \geq r-1,
$$

so $\exp _{2}\left(\tau_{[1,2 r-1]} S^{0}\right) \geq r-1$ as well (in view of Example 2.4).

Let $p$ be an odd prime. We will now give the analogous argument in this case.

Proposition 4.3 We have $\exp _{p}\left(\tau_{[1, n]} S^{0}\right) \geq\lfloor(n-1) /(2 p-2)\rfloor$.

Proof For simplicity, we will work with $B \Sigma_{p}$ (which, implicitly, will be $p$-localized) rather than $B \mathbb{Z} / p$. The $p$-local homology of $B \Sigma_{p}$ is well-known (for the mod $p$ homology from which this can be derived, together with the absence of higher Bocksteins, see [18, Lemmma 1.4]): one has

$$
H_{i}\left(B \Sigma_{p} ; \mathbb{Z}_{(p)}\right) \simeq \begin{cases}\mathbb{Z}_{(p)} & \text { if } i=0, \\ \mathbb{Z} / p & \text { if } i=k(2 p-2)-1, k>0, \\ 0 & \text { otherwise. }\end{cases}
$$

One can thus build a cell decomposition of the (reduced) suspension spectrum $\Sigma^{\infty} B \Sigma_{p}$ with cells in degrees $\equiv 0,-1 \bmod (2 p-2)$ starting in degree $2 p-1$.

Let $k>0$, and consider the $((2 p-2) k)$-skeleton of this complex. We obtain a finite $p$-torsion spectrum $Y_{k}$ equipped with a map

$$
Y_{k} \rightarrow \Sigma^{\infty} B \Sigma_{p}
$$

inducing an isomorphism in $H_{*}\left(\cdot ; \mathbb{Z}_{(p)}\right)$ up to and including degree $k(2 p-2)$. That is, by universal coefficients, $H^{i}\left(Y_{k} ; \mathbb{Z}_{(p)}\right) \simeq \mathbb{Z} / p$ if $i=2 p-2,2(2 p-2), \ldots, k(2 p-2)$, and is zero otherwise.

We now claim

$$
K^{0}\left(Y_{k}\right) \simeq \mathbb{Z} / p^{k}
$$

In order to see this, we use the Atiyah-Hirzebruch spectral sequence (AHSS)

$$
H^{*}\left(Y_{k} ; \mathbb{Z}\right) \Longrightarrow K^{*}\left(Y_{k}\right)
$$


Since the cohomology of $Y_{k}$ is concentrated in even degrees, the AHSS degenerates and we find that $K^{0}\left(Y_{k}\right)$ is a finite $p$-group of length $k$. However, the extension problems are solved by naturality with the map $Y_{k} \rightarrow \Sigma^{\infty} B \Sigma_{p}$, as $\widetilde{K}^{0}\left(B \Sigma_{p}\right) \simeq \mathbb{Z}_{p}$ after $p$-adic completion.

Now $Y_{k}$ is a finite spectrum with cells in degrees $[(2 p-2)-1,(2 p-2) k]$. Let $m=(2 p-2)(k-1)+1$. Then we have, by Lemma 4.1 and $(7)$,

$$
\exp _{p}\left(Y_{k}\right)=\exp _{p}\left(\tau_{[0, m]} S^{0} \wedge Y_{k}\right) \geq k .
$$

Finally, $\exp _{p}\left(H \mathbb{Z} \wedge Y_{k}\right)=1$ since the $p$-local homology of $Y_{k}$ is annihilated by $p$. It follows that $\exp _{p}\left(\tau_{[1, m]} S^{0}\right) \geq k-1$, which is the estimate we wanted if we choose $k$ as large as possible so that $m=(2 p-2)(k-1)+1 \leq n$.

Remark In view of the Kahn-Priddy theorem [16], it is not surprising that the skeleta of classifying spaces of symmetric groups should yield strong lower bounds for torsion in the Postnikov sections of the sphere.

\section{The Hurewicz map}

We next apply our results about the Postnikov sections $\tau_{[1, m]} S^{0}$ to the original question of understanding the exponents in the Hurewicz map. Let $Y$ be a connective spectrum. Then the Hurewicz map is realized as the map in homotopy groups induced by the map of spectra

$$
Y \wedge S^{0} \rightarrow Y \wedge H \mathbb{Z}
$$

whose fiber is $Y \wedge \tau_{[1, \infty]} S^{0}$. As a result of the long exact sequence in homotopy, we find the following result.

Proposition 5.1 Let $Y$ be any connective spectrum.

(a) Suppose $\tau_{[1, n]} S^{0}$ is annihilated by $N$ for some $N>0$. Then any element $x$ in the kernel of the Hurewicz map $\pi_{n}(Y) \rightarrow H_{n}(Y ; \mathbb{Z})$ satisfies $N x=0$.

(b) Suppose $\tau_{[1, n-1]} S^{0}$ is annihilated by $N^{\prime}$ for some $N^{\prime}>0$. Then for any element $y \in H_{n}(Y ; \mathbb{Z}), N^{\prime} y$ is in the image of the Hurewicz map.

The homotopy groups of $X \wedge \tau_{\geq 1} S^{0}$ are classically denoted $\Gamma_{i}(X)$ (and called Whitehead's $\Gamma$-groups). The following argument also appears in, for example, [7, Theorem 6.6], [20, Corollary 4.6], and [10]. 
Proof For the first claim, consider the fiber sequence $Y \wedge \tau_{[1, \infty]} S^{0} \rightarrow Y \rightarrow Y \wedge H \mathbb{Z}$. Any element $x \in \pi_{n}(Y)$ in the kernel of the Hurewicz map lifts to an element $x^{\prime} \in$ $\pi_{n}\left(Y \wedge \tau_{[1, \infty]} S^{0}\right)$. It suffices to show that $N x^{\prime}=0$. But we have an isomorphism

$$
\pi_{n}\left(Y \wedge \tau_{[1, \infty]} S^{0}\right) \simeq \pi_{n}\left(Y \wedge \tau_{[1, n]} S^{0}\right),
$$

and the latter group is annihilated by $N$ by hypothesis (and Example 2.2), so $N x^{\prime}=0$ as desired.

Now fix $y \in H_{n}(Y ; \mathbb{Z})$. In order to show that $N^{\prime} y$ belongs to the image of the Hurewicz map, it suffices to show that it maps to zero via the connective homomorphism into $\pi_{n-1}\left(Y \wedge \tau_{[1, \infty]} S^{0}\right)$. But we have an isomorphism $\pi_{n-1}\left(Y \wedge \tau_{[1, \infty]} S^{0}\right) \simeq$ $\pi_{n-1}\left(Y \wedge \tau_{[1, n-1]} S^{0}\right)$ and this latter group is annihilated by $N^{\prime}$.

Remark One has an evident $p$-local version of Proposition 5.1 for $p$-local spectra if one works instead with $\tau_{[1, n]} S_{(p)}^{0}$.

Proof of Theorem 1.3 The main result on exponents follows now by combining Proposition 5.1 and our upper bound estimates in Theorem 2.8.

It remains to show that the bound is close to being the best possible. This will follow by re-examining our arguments for the lower bounds.

Proof of Proposition 1.4 We start with the prime 2. For this, we use the space $\mathbb{R P}^{2 k}$ and form the endomorphism ring spectrum $Z=\operatorname{Hom}\left(\Sigma^{\infty} \mathbb{R P}^{2 k}, \Sigma^{\infty} \mathbb{R} \mathbb{P}^{2 k}\right) \simeq$ $\Sigma^{\infty} \mathbb{R P}^{k} \wedge \mathbb{D}\left(\Sigma^{\infty} \mathbb{R P}^{2 k}\right)$ where $\mathbb{D}$ denotes Spanier-Whitehead duality. The spectrum $Z$ is not connective, but it is $(1-2 k)$-connective (ie its cells begin in degree $1-2 k$ ). Then we have a class $x \in \pi_{0}(Z)$ representing the identity self-map of $\Sigma^{\infty} \mathbb{R P}^{2 k}$. We know that $x$ has order at least $2^{k}$ (in view of (6)), but that $2 x$ maps to zero under the Hurewicz map since the homology of $Z$ is a sum of copies of $\mathbb{Z} / 2$ in various degrees by the integral Künneth formula, and since the homology of $\mathbb{R P} \mathbb{P}^{2 k}$ is annihilated by 2 . If we replace $Z$ by $\Sigma^{2 k-1} Z$, we obtain a connective spectrum together with a class (the translate of $2 x$ ) in $\pi_{2 k-1}$ of order at least $2^{k-1}$ that maps to zero under the Hurewicz map.

At an odd prime, one carries out the analogous procedure using the spectra $Y_{k}$ used in Proposition 4.3, and (8). One takes $k=r+1$.

Remark We are grateful to Peter May for pointing out the following. Choose $q \geq 0$, and consider the cofiber sequence

$$
C=\tau_{\geq 0} S^{-q} \rightarrow S^{-q} \rightarrow \tau_{<0} S^{-q} .
$$


Choosing $n>0$ and $q$ appropriately, we can find an element in $\pi_{n}(C)=\pi_{n+q}\left(S^{0}\right)$ of large exponent (eg using the image of the $J$-homomorphism), larger than $\exp \left(\tau_{[1, n]} S^{0}\right)$. This element must therefore not be annihilated by the Hurewicz map $\pi_{n}(C) \rightarrow$ $H_{n}(C ; \mathbb{Z})$. Let the image in $H_{n}(C ; \mathbb{Z})$ be $x$. However, the map $H_{n}(C ; \mathbb{Z}) \rightarrow$ $H_{n}\left(S^{-q} ; \mathbb{Z}\right)$ is zero, so $x$ must be in the image of $H_{n+1}\left(\tau_{<0} S^{-q} ; \mathbb{Z}\right)$. This gives interesting and somewhat mysterious examples of homology classes in degree $n$ of a coconnective spectrum.

\section{Applications}

We close the paper by noting a few applications of considering the exponent of the spectrum itself. These are mostly formal and independent of Theorem 2.8, which nevertheless supplies the explicit bounds.

We begin by recovering and improving upon a result from [4] on $k$-invariants.

Theorem 6.1 Let $X$ be a connective spectrum. Then the $n^{\text {th }} k$-invariant $\tau_{\leq n-1} X \rightarrow$ $\Sigma^{n+1} H \pi_{n} X$ is annihilated by $\exp \left(\tau_{[1, n]} S^{0}\right)$.

Proof It suffices to show that $H^{n+1}\left(\tau_{\leq n-1} X ; \pi_{n} X\right)$ is annihilated by $\exp \left(\tau_{[1, n]} S^{0}\right)$. By the universal coefficient theorem (and the fact that the universal coefficient exact sequence splits), it suffices to show that the two abelian groups $H_{n}\left(\tau_{\leq n-1} X ; \mathbb{Z}\right)$ and $H_{n+1}\left(\tau_{\leq n-1} X ; \mathbb{Z}\right)$ are each annihilated by $\exp \left(\tau_{[1, n]} S^{0}\right)$. This follows from the cokernel part of Proposition 5.1 as $\tau_{\leq n-1} X$ has no homotopy in degrees $n$ or $n+1$.

Corollary 6.2 If $X$ is a connective spectrum, then the $n^{\text {th }} k$-invariant of $X$ has $p$-exponent at most $\lceil n / 2\rceil+3$ for $p=2$, and at most $\lceil(n+3) /(2 p-2)\rceil+1$ for $p>2$.

Asymptotically, Corollary 6.2 is stronger than the results of [4], which give $p$-exponent $n-C_{p}$ for $C_{p}$ a constant depending on $p$, as $n \rightarrow \infty$.

Next, we consider a question about the homology of infinite loop spaces.

Theorem 6.3 Let $X$ be an $(m-1)$-connected infinite loop space. Then the kernel of the (unstable) Hurewicz map $\pi_{n}(X) \rightarrow H_{n}(X ; \mathbb{Z})$ is annihilated by $\exp \left(\tau_{[1, n-m]} S^{0}\right)$. Therefore, the $p$-exponent of the kernel is at most $\lceil(n-m) / 2\rceil+3$ for $p=2$, and at most $\lceil(n-m+3) /(2 p-2)\rceil+1$ for $p>2$.

This improves upon (and makes explicit) a result of Beilinson [10], who also considers the cokernel of the map from $\pi_{n}(X)$ to the primitives in $H_{n}(X ; \mathbb{Z})$. 
Proof Without loss of generality, we can assume that $X$ is $n$-truncated. Let $Y$ be the $m$-connective spectrum that deloops $X$. Consider the cofiber sequence

$$
Y \rightarrow \tau_{\leq n-1} Y \rightarrow \Sigma^{n+1} H \pi_{n} Y .
$$

By Theorem 6.1, the $k$-invariant map $\tau_{\leq n-1} Y \rightarrow \Sigma^{n+1} H \pi_{n} Y$ is annihilated by $\exp \left(\tau_{[1, n-m]} S^{0}\right)$. Consider the rotated cofiber sequence

$$
\Sigma^{-1} \tau_{\leq n-1} Y \rightarrow \Sigma^{n} H \pi_{n} Y \rightarrow Y .
$$

Using the natural long exact sequence, we obtain a map

$$
Y \rightarrow \Sigma^{n} H \pi_{n} Y,
$$

which induces multiplication by $\exp \left(\tau_{[1, n-m]} S^{0}\right)$ on $\pi_{n}$. Compare [3, Lemma 4] for this argument.

Delooping, we obtain a map of spaces $\phi: X \rightarrow K\left(\pi_{n} X, n\right)$ which induces multiplication by $\exp \left(\tau_{[1, n-m]} S^{0}\right)$ on $\pi_{n}$. Now we consider the commutative diagram

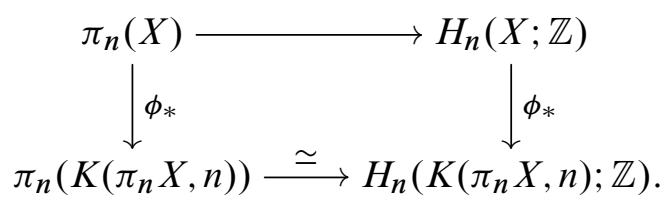

Choose $x \in \pi_{n}(X)$ to be in the kernel of the Hurewicz map; the diagram shows that $\phi_{*}(x)=\exp \left(\tau_{[1, n-m]} S^{0}\right) x=0$, as desired.

Next, we give a more careful statement of Theorem 1.5 (in terms of exponents of Postnikov sections of $S^{0}$ ), and prove it. Note that this result is generally much sharper than Corollary 2.7.

Proposition 6.4 Let $X$ be a $p$-torsion spectrum with homotopy groups concentrated in an interval $[a, b]$ of length $\ell=b-a$. Suppose $p^{k}$ annihilates $\pi_{i}(X)$ for each $i$. Then $\exp _{p}(X) \leq k+\exp _{p}\left(\tau_{[1, \ell]} S^{0}\right)+\exp _{p}\left(\tau_{[1, \ell-1]} S^{0}\right)=k+\ell /(p-1)+O(1)$.

The argument is completely formal with the exception of the equality $\exp _{p}\left(\tau_{[1, \ell]} S^{0}\right)+$ $\exp _{p}\left(\tau_{[1, \ell-1]} S^{0}\right)=\ell /(p-1)+O(1)$. This comparison follows from Theorem 2.8. Proposition 6.4 plus the estimates of Theorem 2.8 yield Theorem 1.5. We note that a simple calculation can make $O(1)$ explicit.

Proof Without loss of generality, we assume $a=0$ so $b=\ell$. We consider the cofiber sequence and diagram

$$
\tau_{[1, \infty]} S^{0} \wedge X \rightarrow X \rightarrow H \mathbb{Z} \wedge X
$$


This induces an exact sequence

$$
\pi_{0} \operatorname{Hom}(H \mathbb{Z} \wedge X, X) \rightarrow \pi_{0} \operatorname{Hom}(X, X) \rightarrow \pi_{0} \operatorname{Hom}\left(\tau_{[1, \infty]} S^{0} \wedge X, X\right) .
$$

Let $R_{1}=p^{\exp _{p}\left(\tau_{[1, b]} S^{0}\right)}$ and $R_{2}=p^{\exp _{p}\left(\tau_{[1, b-1]} S^{0}\right)}$. We will bound the exponents of the terms on either side by $R_{1}$ and $R_{2} p^{k}$ in order to bound the exponent on the group in the middle (which will give a torsion exponent for $X$ ). Note that since $X$ is concentrated in degrees $[0, b]$, one has the following:

$$
\begin{aligned}
\pi_{0} \operatorname{Hom}(H \mathbb{Z} \wedge X, X) & \simeq \pi_{0} \operatorname{Hom}\left(\tau_{\leq b}(H \mathbb{Z} \wedge X), X\right), \\
\pi_{0} \operatorname{Hom}\left(\tau_{[1, \infty]} S^{0} \wedge X, X\right) & \simeq \pi_{0} \operatorname{Hom}\left(\tau_{[1, b]} S^{0} \wedge X, X\right) .
\end{aligned}
$$

We claim first that $\tau_{\leq b}(H \mathbb{Z} \wedge X)$ is annihilated by $R_{2} p^{k}$. To see this, it suffices to show, since $\tau_{\leq b}(H \mathbb{Z} \wedge X)$ is a generalized Eilenberg-MacLane spectrum, that its homotopy groups are each annihilated by $R_{2} p^{k}$. That is, we need to show that each of the homology groups of $X$ is annihilated by $R_{2} p^{k}$. For this, we consider the Hurewicz homomorphism

$$
\pi_{i}(X) \rightarrow H_{i}(X ; \mathbb{Z}) \text { for } i \leq b .
$$

The source is annihilated by $p^{k}$, and Proposition 5.1 implies that the cokernel is annihilated by $R_{2}$. This proves that $H_{i}(X ; \mathbb{Z})$ is annihilated by $R_{2} p^{k}$ for each $i \in[0, b]$. Therefore, (10) is annihilated by $R_{2} p^{k}$.

Next, we claim that $\tau_{[1, b]} S^{0} \wedge X$ is annihilated by $R_{1}$. This is evident by Example 2.4, because $\tau_{[1, b]} S^{0}$ is. Thus, (11) is annihilated by $R_{1}$.

Putting everything together, we obtain the desired torsion bounds on the ends of (9), so the middle term is annihilated by $R_{1} R_{2} p^{k}$, and we are done.

Finally, we show that our results have applications to exponent theorems in equivariant stable homotopy theory. We begin by noting a useful example on the stable homotopy of classifying spaces.

Example 6.5 Let $G$ be a finite group and let $\Sigma^{\infty} B G$ be the reduced suspension spectrum of the classifying $B G$. Then for any $n$, the abelian group $\pi_{n}\left(\Sigma^{\infty} B G\right)$ is annihilated by $|G| \exp \left(\tau_{[1, n]} S^{0}\right)$. This follows from Proposition 5.1 since the integral homology of $B G$ is annihilated by $|G|$. In fact, we obtain that the spectrum $\tau_{[1, n]} B G$ is annihilated by $|G| \exp \left(\tau_{[1, n]} S^{0}\right)$. We do not know if the growth rate of $\exp \left(\tau_{[1, n]} B G\right)$ is in general comparable to this.

Let $G$ be a finite group, and consider the homotopy theory $\mathcal{S}_{G}$ of genuine $G$-equivariant spectra. The symmetric monoidal category $\mathcal{S}_{G}$ has a unit object, the equivariant sphere 
$S^{0}$. We will be interested in exponents for the equivariant stable stems $\pi_{n, G}\left(S^{0}\right)=$ $\pi_{0} \operatorname{Hom}_{\mathcal{S}_{G}}\left(S^{n}, S^{0}\right)$. More generally, we will replace the target $S^{0}$ by a representation sphere $S^{V}$, for $V$ a finite-dimensional real representation of $G$. In this case, we will write $\pi_{n, G}\left(S^{V}\right)=\operatorname{Hom}_{\mathcal{S}_{G}}\left(S^{n}, S^{V}\right)$. For a subgroup $H \subset G$, we will write $W H=N_{G}(H) / H$ for the Weyl group.

Theorem 6.6 Let $V$ be a finite-dimensional $G$-representation. Suppose $n$ is not equal to the dimension $\operatorname{dim} V^{H}$ for any subgroup $H \subset G$. Then the abelian group $\pi_{n, G}\left(S^{V}\right)$ is annihilated by the least common multiple of $\left\{|W H| \exp \left(\tau_{\left[1, n-\operatorname{dim} V^{H}\right]} S^{0}\right)\right\}$ as $H \subset G$ ranges over all the subgroups with $\operatorname{dim} V^{H}<n$. In particular, the $p$-exponent of $\pi_{n, G}\left(S^{V}\right)$ is at most

$$
\begin{aligned}
\exp _{p}\left(\pi_{n, G}\left(S^{V}\right)\right) \leq & \max _{H \subset G, \operatorname{dim} V^{H}<n}\left(v_{p}(|W H|)+\exp _{p}\left(\tau_{\left[1, n-\operatorname{dim} V^{H}\right]} S^{0}\right)\right) \\
& =\max _{H, \operatorname{dim} V^{H}<n}\left(v_{p}(|W H|)+\frac{n-\operatorname{dim} V^{H}}{2 p-2}\right)+O(1),
\end{aligned}
$$

where $v_{p}$ denotes the $p$-adic valuation.

Remark The least common multiple simplifies to $|G| \exp \left(\tau_{[1, n-\operatorname{dim} V]} S^{0}\right)$ when $n>$ $\operatorname{dim} V$.

Proof This follows from the Segal-tom Dieck splitting [13], which implies that

$$
\pi_{n, G}\left(S^{V}\right)=\bigoplus_{H} \pi_{n}\left(\left(\Sigma^{\infty} S^{V^{H}}\right)_{h W_{H}}\right),
$$

where $H$ ranges over a system of conjugacy classes of subgroups of $G$. When $V$ is the trivial representation, we can apply Example 6.5 to conclude.

In general, we have that $\left(\Sigma^{\infty} S^{V^{H}}\right)_{h W_{H}}$ is $\operatorname{dim} V^{H}$-connective. Moreover, the homology $H_{*}\left(S^{V^{H}} ; \mathbb{Z}\right)$ is concentrated in dimension $\operatorname{dim} V^{H}$, so it follows that for $n>$ $\operatorname{dim} V^{H}, H_{n}\left(\left(\Sigma^{\infty} S^{V^{H}}\right)_{h W H} ; \mathbb{Z}\right)$ is annihilated by the order of $W H$. For $n<\operatorname{dim} V^{H}$, there is no contribution in homotopy from $\left(\Sigma^{\infty} S^{V^{H}}\right)_{h W_{H}}$. Applying Proposition 5.1 and Theorem 2.8, we obtain the desired exponent result.

In equivariant stable homotopy theory, one is more generally interested in maps $S^{W} \rightarrow$ $S^{V}$ where $W$ and $V$ are orthogonal representations of $G$. Unfortunately, the method of Theorem 6.6 does not seem to give anything unless $W$ is very small relative to $V$, in which case one can use a cell decomposition of $S^{W}$ and apply Theorem 6.6 to the individual cells. 


\section{References}

[1] J F Adams, Stable homotopy theory, 2nd revised edition, Lecture Notes Math. 3, Springer, Berlin (1966) MR0196742

[2] J F Adams, Stable homotopy and generalised homology, Univ. Chicago Press, Chicago, IL (1974) MR0402720

[3] D Arlettaz, On the homology of the special linear group over a number field, Comment. Math. Helv. 61 (1986) 556-564 MR870706

[4] D Arlettaz, On the $k$-invariants of iterated loop spaces, Proc. Roy. Soc. Edinburgh Sect. A 110 (1988) 343-350 MR974750

[5] D Arlettaz, Universal bounds for the exponent of stable homotopy groups, Topology Appl. 38 (1991) 255-261 MR1098905

[6] D Arlettaz, The exponent of the homotopy groups of Moore spectra and the stable Hurewicz homomorphism, Canad. J. Math. 48 (1996) 483-495 MR1402323

[7] D Arlettaz, Algebraic K-theory of rings from a topological viewpoint, Publ. Mat. 44 (2000) 3-84 MR1775748

[8] D Arlettaz, The generalized Boardman homomorphisms, Cent. Eur. J. Math. 2 (2004) 50-56 MR2041667

[9] MF Atiyah, K-theory, Benjamin, New York (1967) MR0224083

[10] A Beilinson, Relative continuous K-theory and cyclic homology, Münster J. Math. 7 (2014) 51-81 MR3271239

[11] F R Cohen, J C Moore, J A Neisendorfer, The double suspension and exponents of the homotopy groups of spheres, Ann. of Math. 110 (1979) 549-565 MR554384

[12] E S Devinatz, M J Hopkins, J H Smith, Nilpotence and stable homotopy theory, I, Ann. of Math. 128 (1988) 207-241 MR960945

[13] T tom Dieck, Orbittypen und äquivariante Homologie, II, Arch. Math. (Basel) 26 (1975) 650-662 MR0436177

[14] H-W Henn, On the growth of homotopy groups, Manuscripta Math. 56 (1986) 235-245 MR850372

[15] M J Hopkins, The mathematical work of Douglas C Ravenel, Homology, Homotopy Appl. 10 (2008) 1-13 MR2475614

[16] D S Kahn, S B Priddy, The transfer and stable homotopy theory, Math. Proc. Cambridge Philos. Soc. 83 (1978) 103-111 MR0464230

[17] A Liulevicius, Zeroes of the cohomology of the Steenrod algebra, Proc. Amer. Math. Soc. 14 (1963) 972-976 MR0157383

[18] J P May, A general algebraic approach to Steenrod operations, from: "The Steenrod algebra and its applications", (F P Peterson, editor), Lecture Notes in Mathematics 168, Springer, Berlin (1970) 153-231 MR0281196 
[19] J McCleary, A user's guide to spectral sequences, 2nd edition, Cambridge Studies Adv. Math. 58, Cambridge Univ. Press, Cambride (2001) MR1793722

[20] J Scherer, Exponents for high-dimensional gamma groups, Exposition. Math. 13 (1995) 455-468 MR1362871

Department of Mathematics, University of California,

970 Evans Hall, Berkeley, CA 94720, USA

amathew@math . harvard. edu

http://math.harvard.edu/ amathew/

Received: 26 March 2015 Revised: 29 July 2015 
